\title{
Editorial: Actinobacteria: Recent Trends in Genomics, Omics Study and Discovery of Novel Natural Products
}

\author{
Ajit Kumar Passari and Sergio Sánchez* \\ Departamento de Biología Molecular y Biotecnología, Instituto de Investigaciones Biomédicas, Universidad Nacional \\ Autónoma de México, México, Mexico
}

Keywords: actinobacteria, genomics, natural bioactive compounds, transcriptomics, metabolomics

\section{Editorial on the Research Topic}

Actinobacteria: Recent Trends in Genomics, Omics Study and Discovery of Novel Natural Products

Bacteria, particularly actinobacteria, and fungi, among the microorganisms that generate the constituents above, produce a varied array of small bioactive molecules with significant potential for use in medicine (O'Brien and Wright, 2011). Antibiotics, pigments, growth hormones, anticancer drugs, and other microbial secondary metabolites are not essential for microbe growth and development, but they have shown considerable promise for human and animal health (Sánchez and Demain, 2014). These secondary metabolites are formed mainly by the activation of cryptic gene clusters that are inactive under certain conditions; consequently, increasing the expression

OPEN ACCESS

Edited by:

George Tsiamis,

University of Patras, Greece

Reviewed by:

Vassiliki Karapapa,

Municipality of Agrinio, Greece

*Correspondence:

Sergio Sánchez

sersan@biomedicas.unam.mx

Specialty section:

This article was submitted to Systems Microbiology,

a section of the journal

Frontiers in Microbiology

Received: 22 October 2021 Accepted: 15 November 2021 Published: 08 December 2021

Citation:

Passari AK and Sánchez S (2021)

Editorial: Actinobacteria: Recent Trends in Genomics, Omics Study and Discovery of Novel Natural Products. Front. Microbiol. 12:799737. doi: 10.3389/fmicb.2021.799737 of these clusters could assist in exploiting microorganism's chemical variability (Pettit, 2011; Guzmán-Trampe et al., 2017; Xu et al., 2019).

Actinobacteria are Gram-positive microbes with rod-shaped or filamentous morphology. They inhabit diverse environments and have a high $\mathrm{G}+\mathrm{C}$ content in their DNA. Actinobacteria have had a significant influence on human health and well-being (Demain and Sanchez, 2009). Actinobacteria have been obtained from various ecosystems like saline soil, freshwater sediments, sponges, animal and human guts, medicinal plants, deep forests, hot springs, etc. Due to their various ecological functions have multiple applications in agriculture, biomedical, industrial, and pharmaceutical, apart from antibiotic production (Demain et al., 2019). The application of omics tools (genomics, proteomics, transcriptomics, metabolomics, and NGS studies) in actinobacteria has impacted novel bioactive compounds detection and production (Orsi et al., 2016; Singh et al.). Still, the physiological functions of actinobacteria and their ecological interactions await further investigation. The publication of the complete genome sequence of the actinobacteria model Streptomyces coelicolor A3 (2) stimulated the development of computational resources. These include bioinformatics tools like "Antibiotics and Secondary Metabolites Analysis SHell (antiSMASH)" and the "Prediction Informatics for Secondary Metabolomes (PRISM)" (MartínezKlimova et al., 2016). With these instruments, many researchers have discovered the presence of "cryptic" or silent biosynthetic gene clusters (Takagi and Shin-ya, 2011; Harrison and Studholme, 2014). Therefore, many researchers have used the CRISPR/Cas9 system as a tool to elicit the production of unknown secondary metabolites by (a) either knock-in genes to activate silent biosynthetic gene clusters or (b) deleting repressor genes (Jia et al., 2017; Zhang et al., 2017).

Under the title "Actinobacteria: Recent Trends in Genomics, Omics Study and Discovery of Novel Natural Products," a total of six articles were published, covering a variety of topics orbiting around actinobacteria. The special issue includes three reviews and three research articles on actinobacteria dealing with: antibiotic production, ecological modifications, transcriptomic 
profiling, gene modulation by post-translational modifications, and the discovery of novel secondary metabolites with their bioactive properties. Thus, a survey by Droste et al. emphasized that transcriptome and proteome data are valuable for improving the annotation of the strain Streptomyces lividans TK24 genome. Strain TK24 could be used to examine secondary metabolite gene clusters analysis. Martin et al. came out with the role of posttranslational modifications in bacterial metabolism modulation, especially on their effects on secondary metabolite biosynthesis. Kim et al. have focused on enhanced ohmyungsamycin A (OMS) production through adenylation (A2) domain engineered Streptomyces strains under the optimized culture conditions. Ohmyungsamycin A showed significant activity against Mycobacterium tuberculosis and human cancer cells. Hesketh et al. pointed out that results of chemotranscriptomic profiling defined the specific signatures of the glycopeptide antibiotics dalbavancin, vancomycin, and chlorobiphenylvancomycin tested at the bacterium's transcriptional response level. Singh et al. described that actinobacteria are well-known for producing diverse secondary metabolites and supported the genomic approach developments as a gateway for examining and manipulating novel antibiotic gene clusters. Kalam et al. described the influence of the phylum acidobacteria in vital ecological processes. They proposed exploring these bacteria's genetic attributes of understand better the functions and ecological significance in the soil-plant environment.

We are enchanted to present this research topic in Frontiers in Microbiology. We hope this special issue will be exciting and

\section{REFERENCES}

Demain, A. L., Gómez-Ortiz, B., Ruiz-Villafán, B., Rodríguez-Sanoja, R., and Sánchez, S. (2019). Recent findings of molecules with anti-infective activity: screening of non-conventional sources. Curr. Opin. Pharm. 48, 40-47. doi: 10.1016/j.coph.2019.04.003

Demain, A. L., and Sanchez, S. (2009). Microbial drug discovery: 80 years of progress. J. Antibiot. 62, 5-16. doi: 10.1038/ja.2008.16

Guzmán-Trampe, S., Manzo-Ruiz, M., Ceapa, C. D., and Sánchez, S. (2017). Synthetic biology era: improving antibiotic's world. Biochem. Pharmacol. 134, 99-113. doi: 10.1016/j.bcp.2017.01.015

Harrison, J., and Studholme, D. J. (2014). Recently published Streptomyces genome sequences. Microb. Biotechnol. 7, 373-380. doi: 10.1111/1751-7915.12143

Jia, H., Zhang, L., Wang, T., Han, J., Tang, H., and Zhang, L. (2017). Development of a CRISPR/Cas9-mediated gene-editing tool in Streptomyces rimosus. Microbiology 163, 1148-1155. doi: 10.1099/mic.0.000501

Martínez-Klimova, E., Centeno-Leija, S., and Sánchez, S. (2016). "Chapter 1: The impact of genome-mining in the development of new antiinfectives," in Frontiers in Clinical Drug Research-Anti-Infectives, Vol. 3, ed A. U. Rahman (Sharjah: Bentham Science Publishers), 3-54. doi: $10.2174 / 97816080585491140101$

O'Brien, J., and Wright, G. D. (2011). An ecological perspective of microbial secondary metabolism. Curr. Opin. Biotechnol. 22, 552-558. doi: 10.1016/j.copbio.2011.03.010

Orsi, W. D., Smith, J. M., Liu, S., Liu, Z., Sakamoto, C. M., Wilken, S., et al. (2016). Diverse, uncultivated bacteria and archaea underlying the cycling of dissolved protein in the ocean. ISME J. 10, 2158-2173. doi: 10.1038/ismej.2 016.20

Pettit, R. K. (2011). Small-molecule elicitation of microbial secondary metabolites. Microb. Biotechnol. 4, 471-478. doi: 10.1111/j.1751-7915.2010.0 0196.x beneficial to the journal's readers and spread the information of actinobacteria importance in diverse areas. The knowledge accessible above is promising but still limited. Finally, we acknowledge all contributors, totaling 40 authors, for the relevant scientific information within the research articles and reviews compiled in this issue. We are pretty sure that the information covered and presented will be fascinating and convenient for the readers and can be the idea for the investigation on "Actinobacteria: Genomic approach for the production of Natural Products."

\section{AUTHOR CONTRIBUTIONS}

AP and SS organized this topic and wrote the editorial article for publication. All authors contributed to the article and approved the submitted version.

\section{ACKNOWLEDGMENTS}

The authors are thankful to the DGAPA, UNAM grant IN205519 for their grateful support, which allowed them to complete the postdoctoral research work of AP. Part of this work was supported by the CONACYT Grant S-9143 and the NUATEI program from Instituto de Investigaciones Biomédicas, UNAM. AP also received the Sistema Nacional de Investigadores (SNI) level I from Conacyt (979132) under Gobierno de México. We are thankful to CA Shalu Agrawal, Delhi, India, for the manuscript's critical reading and language editing.

Sánchez, S., and Demain, A. L. (2014). "Valuable products from microbes," in Microbes in Process, eds G. Neelam and A. Abhinav (New York, NY: Nova Scientific Publishers, Inc., USA), 23-57.

Takagi, M., and Shin-ya, K. (2011). New species of actinomycetes do not always produce new compounds with high frequency. J. Antibiot. (Tokyo). 64, 699-701. doi: 10.1038/ja.2011.66

Xu, F., Wu, Y., Zhang, C., Davis, K. M., Moon, K., Bushin, L. B., et al. (2019). A genetics-free method for high-throughput discovery of cryptic microbial metabolites. Nat. Chem. Biol. 15, 161-168. doi: 10.1038/s41589-018-0193-2

Zhang, M. M., Wong, F. T., Wang, Y., Luo, S., Lim, Y. H., Heng, E., et al. (2017). CRISPR-Cas9 strategy for activation of silent Streptomyces biosynthetic gene clusters. Nat. Chem. Biol. 13, 607-609. doi: 10.1038/nchembio.2341

Conflict of Interest: The authors declare that the research was conducted in the absence of any commercial or financial relationships that could be construed as a potential conflict of interest.

Publisher's Note: All claims expressed in this article are solely those of the authors and do not necessarily represent those of their affiliated organizations, or those of the publisher, the editors and the reviewers. Any product that may be evaluated in this article, or claim that may be made by its manufacturer, is not guaranteed or endorsed by the publisher.

Copyright (๑) 2021 Passari and Sánchez. This is an open-access article distributed under the terms of the Creative Commons Attribution License (CC BY). The use, distribution or reproduction in other forums is permitted, provided the original author(s) and the copyright owner(s) are credited and that the original publication in this journal is cited, in accordance with accepted academic practice. No use, distribution or reproduction is permitted which does not comply with these terms. 